\title{
Experimental methodology for the measurement of plasticity on metals at high strain-rates
}

\author{
Alexander Sancho ${ }^{1, *}$, Mike J. Cox ${ }^{1,2}$, Giles Aldrich-Smith ${ }^{2}$, Tim Cartwright ${ }^{2}$, Catrin M. Davies ${ }^{1}$, Paul A. Hooper ${ }^{1}$, and \\ John P. Dear ${ }^{1}$
}

${ }^{1}$ Imperial College London, Department of Mechanical Engineering, SW7 2AZ, London, United Kingdom

${ }^{2}$ AWE Plc, Materials Science, RG7 4PR, Aldermaston, Reading, United Kingdom

\begin{abstract}
An experimental methodology has been developed for the tensile characterisation of ductile isotropic metals at high strain-rate. This study includes the region beyond plastic instability or necking, which is rarely analysed for conventional applications. The research explores an imaging technique used to track the geometry of the specimen during tensile tests and calculate true local values of stress and strain by applying Bridgman theory [1]. To improve the quality of the images taken at high strain-rate an in-situ high speed shadowgraph technique has been developed, and to obtain better results from the images a sub-pixel accuracy edge detection algorithm has been implemented. The technique has been applied to an austenitic stainless steel. Its tensile behaviour has been assessed by testing round samples at strain-rates ranging from quasi-static to $\sim 10^{3} \mathrm{~s}^{-1}$. The results obtained with the proposed methodology have been validated by comparison with more conventional techniques such as video-extensometer and digital image correlation in the pre-necking region and good performance even at the highest strain-rate tested has been proved.
\end{abstract}

\section{Introduction}

The experimental measurement of tensile properties of metals is one of the most extended tests in materials engineering. Nevertheless, the proper characterisation of materials beyond plastic instability or necking is rarely studied due to the lack of interest in the plastic region for conventional applications. The aim of this work is to establish a set of experiments that can be applied for the tensile characterisation of metals at any deformation strain-rate. The results obtained can be used for improved plasticity models calibration.

Many authors have addressed the tensile characterisation of metals at high strain-rate being some examples the work done by Johnson and Cook on copper, iron and steel [2-3], Stout and Follansbee on austenitic steel [4], Smerd et al. on aluminium sheet [5] and El-Magd and Abouridouane on aluminium, magnesium and titanium alloys [6]. The experimental data obtained from these results is generally used to calibrate plasticity models that include the effects of strain-rate on the yield and hardening behaviour. Some of the most commonly used models for this purpose are those developed by Johnson and Cook [2], Zerilli and Armstrong [7], Bai and Wierzbicki [8] or the MTS (mechanical threshold stress) model [9].

Most of the available experimental studies use tensile or compressive split Hopkinson bar method or Taylor technique, which are designed to be used at extremely high strain-rates $\left(10^{4}-10^{6} \mathrm{~s}^{-1}\right)$. The present research focuses on slightly lower rates $\left(10^{2}-10^{4} \mathrm{~s}^{-1}\right)$ using a servo-hydraulic testing machine. These conditions are not so commonly studied and this research aims at providing a methodology for those intermediate strainrates. However, the main improvement of the proposed method compared to other available techniques is that it provides results beyond the point of instability and up to fracture. While other methods ignore the final region of localisation and necking of the material, the proposed experiment is able to provide true stress and strain measurements along the whole plastic region.

In the present paper, the methodology proposed for true local stress and true local strain measurement is firstly presented. Experimental results applying the technique and validation by comparison with other methods are then presented. Finally, some conclusions about the results obtained and the applicability of the methodology are discussed.

\section{Methodology}

\subsection{Material and specimens}

The material investigated in this research is stainless steel $304 \mathrm{~L}$, an austenitic steel with low carbon (max. $0.03 \%$ ) and high chromium (min. 18\%) and nickel (min. $8 \%$ ) contents. The material was provided as drawn round bar that was subsequently machined to produce the required test specimens. A conventional round bar specimen geometry $(\mathrm{RB})$ has been used to characterize the plastic behaviour. The specimens tested in quasi-

* Corresponding author: a.sancho15@,imperial.ac.uk 
static conditions present the same geometry as those used for high strain-rate but the latter have been scaled down in order to accommodate the maximum load to the capacity of the testing machine used. The diameter of the quasi-static specimens is $6 \mathrm{~mm}$ and that of the high strain-rate samples is $3.5 \mathrm{~mm}$.

\subsection{Test setup}

\subsubsection{High speed test equipment}

All the high speed tensile tests have been conducted in a VHS Instron servo-hydraulic test machine capable of running velocities up to $20 \mathrm{~m} / \mathrm{s}$. A diagram explaining the setup of the experiment is presented in Figure 1. A lost-motion device has been used to avoid applying load on the specimen before a constant velocity is achieved. The slack rod inside the actuator piston applies load to the specimen only after the latter has finished the acceleration process.

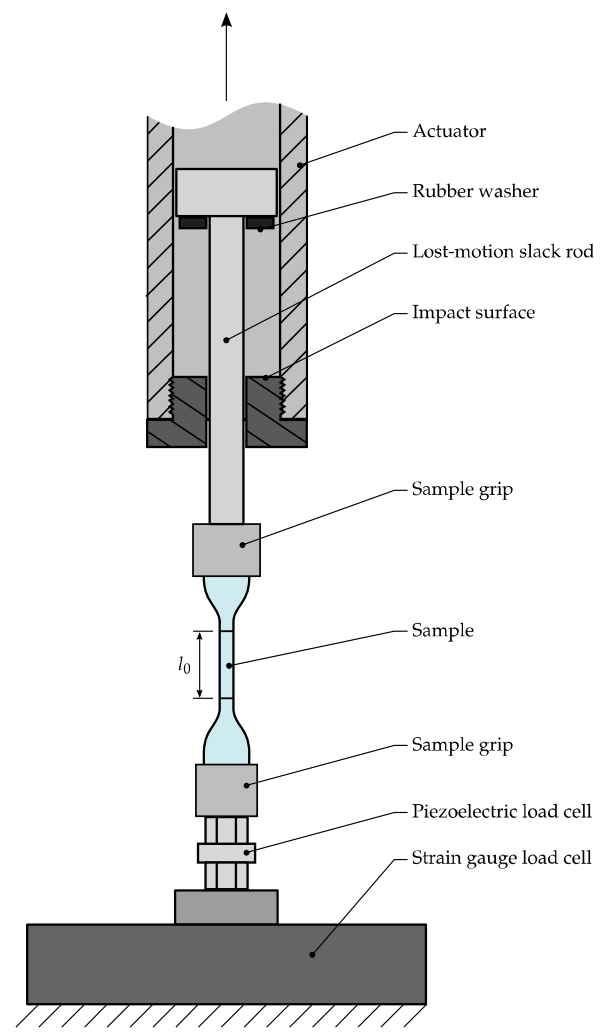

Fig. 1. High speed tensile tests experiment setup.

A piezoelectric PCB222 load cell has been used to measure the load. It has been placed right behind the specimen to obtain the smoothest measurements possible avoiding the ringing produced by joints and threads. Regarding the strain measurement a non-contact method is desired at high speed, therefore, a Phantom V1610 high speed camera has been used to record images of the test in combination with the processing method described below.

\subsubsection{High speed in-situ shadowgraph test}

The test consists of recording images of the specimen and extracting its external shape or edges to obtain the evolution of two geometrical parameters: the radius of the minimum cross-section $(a)$ and the radius of curvature of the neck $(\rho)$. These parameters can be observed in Figure 2 in an example of one the images taken during a quasi-static test.

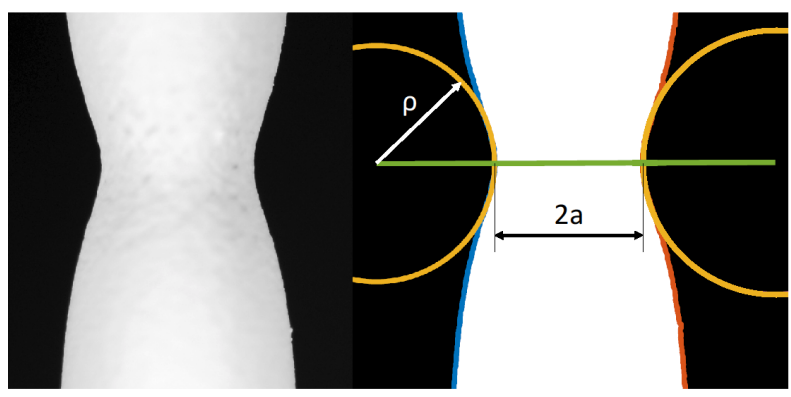

Fig. 2. Images processing for the extraction of geometrical parameters of the neck.

Nevertheless, obtaining images of the samples of sufficient quality at high speed can be challenging. In order to increase the contrast of the images compared to the dark background, specimens are generally painted in white colour and lights used. However, at high speed the paint is not able to follow the fast deformation of the specimen and detaches from it shattering as observed in Figure $3 b$, where it is depicted that the problem becomes even worse during necking, which is the region of interest of this research.

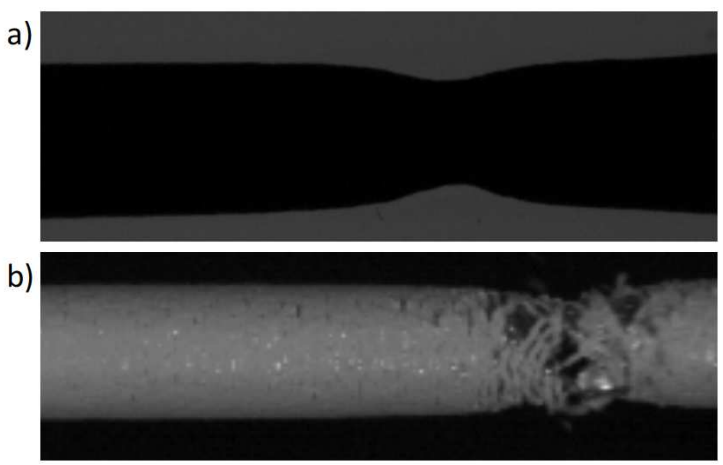

Fig. 3. Image of specimen necking during high speed tensile test using a) the shadowgraph technique and b) conventional pictures of the painted surface of the specimen.

To overcome this difficulty an alternative setup in which the specimen is not painted has been developed. A picture of the test setup is presented in Figure 4. For this test only one light is placed behind the specimen and covered with diffusing paper. The shadow produced by the specimen is recorded by a high speed camera located exactly in the front of the sample and the light. The working principle is the same as that of a shadowgraph but applied during a tensile test at high speed. Figure $3 \mathrm{a}$ shows a picture of the neck obtained using this method where the quality improvement compared to Figure $3 b$ is highlighted. 


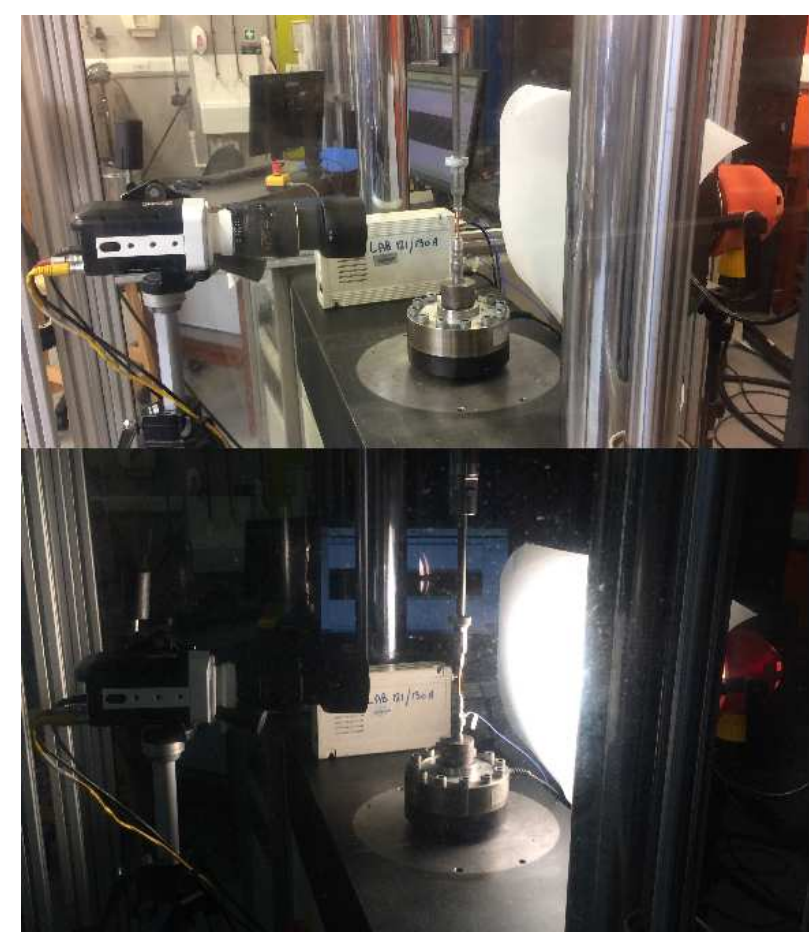

Fig. 4. High speed in-situ shadowgraph test setup.

\subsubsection{Video-extensometer and DIC validation tests}

For comparison with the proposed method other techniques have been applied to the same material and specimens. Since traditional clip-gauge extensometers cannot easily be attached to specimens deformed at high speed, video-extensometers are generally used as an alternative. The measurement taken is the distance between two points of the gauge length in both cases. Engineering strain can be calculated from this relative displacement and engineering stress from the load cell load. In this research two horizontal lines have been painted in the gauge length of the specimen (Figure 5) and tracked with the high speed camera. These have been later post-processed applying a thresholding algorithm to track the position of the lines.

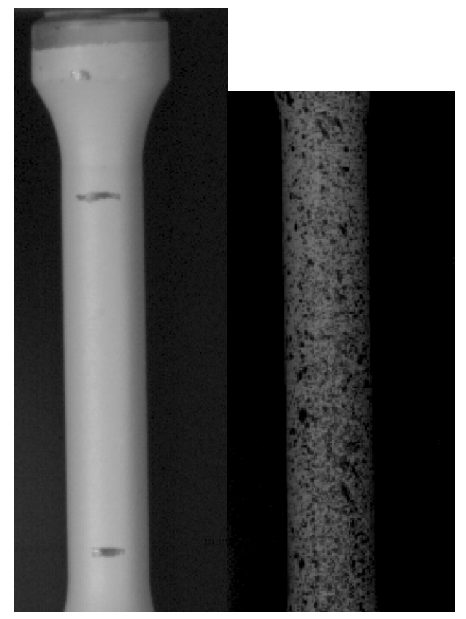

Fig. 5. Samples preparation for video-extensometer and DIC tensile experiments.
2D digital image correlation (DIC) has also been used to calculate the strain on the specimens. This method gives full field information of the surface of the specimen instead of just point-to-point 1D strain, nonetheless, in this research the focus is only on the comparison of strain calculation with other techniques and not the full strain contours. A speckle pattern has been painted on the surface of the specimens as shown in Figure 5 and the images recorded with the high speed camera have been post-processed using ARAMIS software from GOM GmbH.

\subsection{Test post-processing}

Once the tensile tests have been performed the images have been post-processed and values of $a$ and $\rho$ extracted from each picture as observed in Figure 2. The edge extraction algorithm used is explained below as well as the equations used for the calculation of stress and strain using the geometry of the sample.

\subsubsection{Sub-pixel accuracy algorithm}

When images of high resolution are available a simple thresholding algorithm can be used to extract edges from raw images. This process is explained in Figure 6 and consists of transforming the picture from grey scale to pure black and white by defining a threshold grey scale value. In 8-bit black and white images each pixel presents a value that ranges from 0 (pure white) to 255 (pure black); the selected threshold value defines the edge of the specimen. The difficulty lies in obtaining pictures of sufficient resolution to avoid obtaining a stepped edge. This is particularly challenging in high speed tests where resolution is generally lowered in order to increase the frame rate.

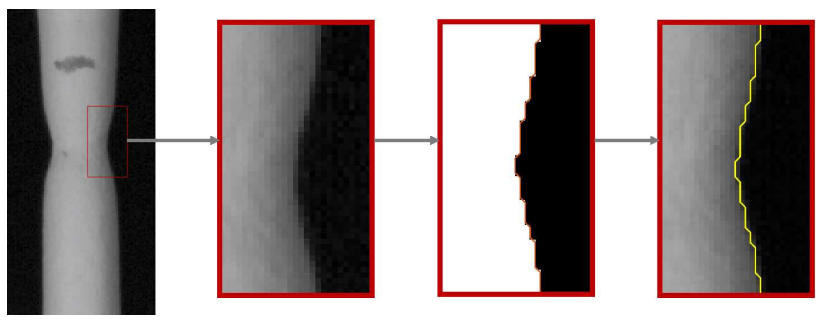

Fig. 6. Thresholding image processing method for specimen edge extraction.

To improve the quality of the extracted edges and the subsequent stress analysis a sub-pixel accuracy edge detection algorithm has been implemented instead. This algorithm is explained in Figure 7 and is able to extract smooth edges from low quality images since it can locate edges within pixels instead of only on their boundaries. The algorithm was proposed by Trujillo-Pino et al. [10] and was originally developed for high precision edge detection in medical images. Instead of analysing each pixel individually this method considers the neighbouring region to locate the edge. The intensity value (grey scale value) of the neighbouring pixels is 
used to estimate the position, orientation and curvature of the edge within the pixel.

Figures 6 and 7 have been obtained using the same raw picture and the quality improvement of the extracted edge when the sub-pixel accuracy method is used can clearly be observed in the images on the left.

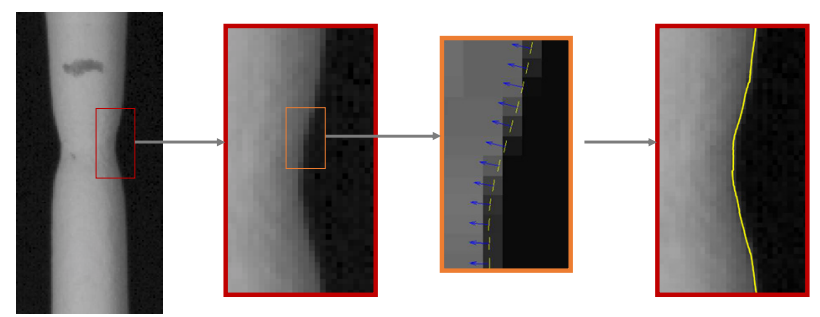

Fig.7. Sub-pixel accuracy image processing method for specimen edge extraction.

\subsubsection{True stress and strain calculation}

Before necking starts the deformation is uniform and the classic approach for true stress and true strain calculation from experimentally obtained stress and strain can be used following Equations 1 and 2:

$$
\begin{aligned}
& \varepsilon_{T}=\ln \left(1+\varepsilon_{e}\right) \\
& \sigma_{T}=\sigma_{e}\left(1+\varepsilon_{e}\right)
\end{aligned}
$$

where $\sigma_{e}$ and $\varepsilon_{e}$ are the engineering values of stress and strain and $\sigma_{T}$ and $\varepsilon_{T}$ the true values.

Nevertheless, the assumption of uniform deformation does not stand once localisation and necking begins and this processing method is no longer valid. Bridgman [1] derived equations based on the changes in geometry of the specimen to calculate the local true stress and true strain in the centre of the neck. Equations 3 and 4 represent a summary of the relationships obtained from that derivation:

$$
\begin{gathered}
\varepsilon_{z z, a v}=2 \ln \left(\frac{a_{0}}{a}\right) \\
\sigma_{z z, M A X}=\frac{\sigma_{z z, a v}}{\left(1+\frac{2 \rho}{a}\right) \ln \left(1+\frac{a}{2 \rho}\right)}\left(1+\ln \left(1+\frac{a}{2 \rho}\right)\right)
\end{gathered}
$$

where $\sigma_{z z, a v}$ and $\varepsilon_{z z, a v}$ are the average stress and strain across the cross-section in axial direction, $\sigma_{z z, M A X}$ the maximum stress in axial direction, $a_{0}$ the initial specimen radius, $a$ the current specimen radius and $\rho$ the current neck radius of curvature.

In this research Equations 3 and 4 have been used to obtain true stress and strain from the measured values of $a$ and $\rho$ following the proposed methodology. Equations 1 and 2 have been used to post-process the videoextensometer and DIC tests in the region of uniform deformation and therefore validate the method.

\section{Experimental results}

All the tests have been run at room temperature conditions and velocities of $1 \mathrm{~m} / \mathrm{s}$ and $12 \mathrm{~m} / \mathrm{s}$. Quasi-static results $\left(\mathrm{v}=2 \times 10^{-5} \mathrm{~m} / \mathrm{s}\right)$ have also been included for comparison purposes. The true equivalent stress - strain results for the tests performed at $1 \mathrm{~m} / \mathrm{s}$ are presented in Figure 8 . The evolution of strain-rate throughout the tests is also represented in the figure. As observed, the results of the tests post-processed with the videoextensometer and DIC techniques coincide with those that include neck correction at the beginning of the tests, validating the performance of the proposed technique in that region. When necking starts the video extensometer and DIC results are not representative though, while the proposed method shows the real increase in local stress and strain after necking and before fracture.

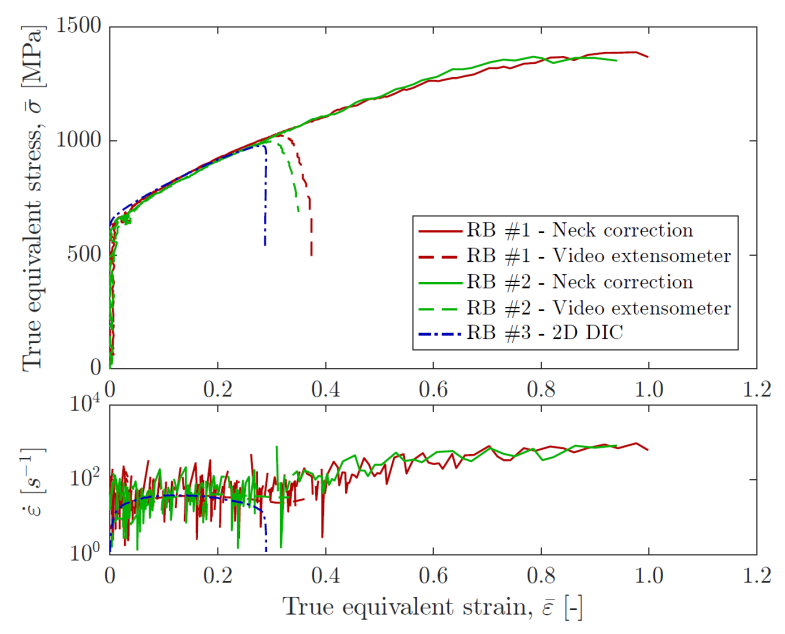

Fig. 8. Comparison of true equivalent stress vs. true equivalent strain at $1 \mathrm{~m} / \mathrm{s}$ speed using the proposed methodology and other techniques (video extensometer and DIC). Strain-rate vs. true equivalent strain curves are also represented.

Regarding the effect of applying a thresholding or a sub-pixel accuracy algorithm, in Figure 9 a comparison of the true equivalent stress - strain obtained using both methods has been plotted also for the $1 \mathrm{~m} / \mathrm{s}$ test. As observed, both curves follow the same trend but the stepped edges obtained from the images with the thresholding technique lead to inaccurate values of the neck curvature, and therefore, a saw-tooth shape in the stress-strain curve.

Figure 10 presents the effect of strain-rate on true equivalent stress - strain of stainless steel 304L. The insitu shadowgraph experiment with sub-pixel accuracy edge detection post-processing algorithm has been used to obtain the results at $1 \mathrm{~m} / \mathrm{s}$ and $12 \mathrm{~m} / \mathrm{s}$. The quasi-static tests have also been processed with the imaging method described in this paper, but a thresholding algorithm has been sufficient to obtain accurate results in that case. The results follow the expected trend with yield and flow stresses increasing and fracture strain decreasing for higher strain-rates. 


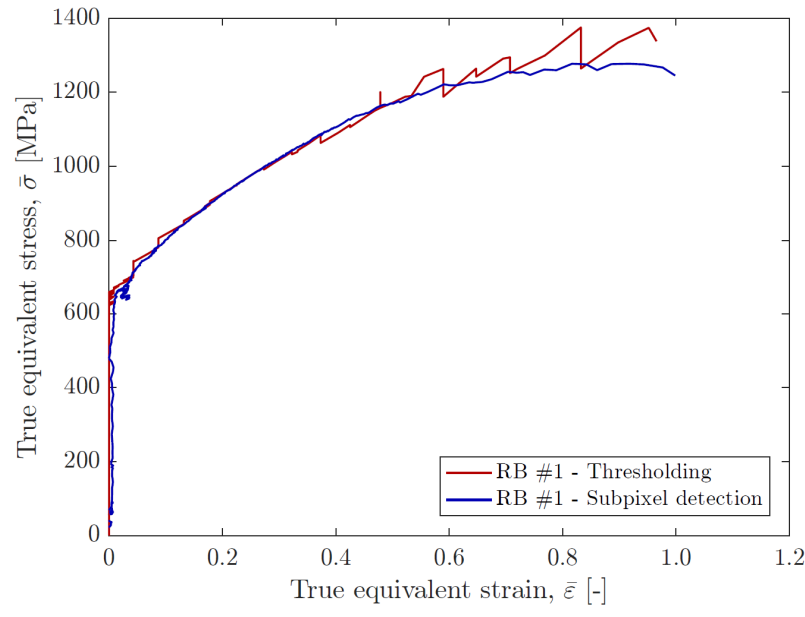

Fig. 9. Comparison of true equivalent stress vs. true equivalent strain using the thresholding and sub-pixel edge detection algorithms.

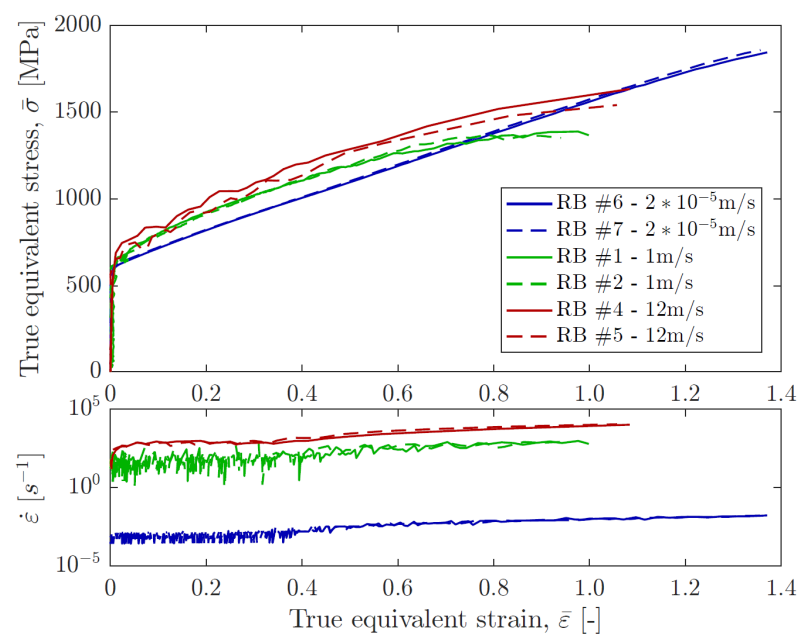

Fig. 10. True equivalent stress vs. true equivalent strain behaviour of stainless steel 304L for different strain-rates using the proposed methodology. Strain-rate vs. true equivalent strain curves are also represented.

\section{Conclusions}

A methodology for the experimental evaluation of true stress and true strain including the local effects of necking at high strain-rate has been proposed. To overcome the challenges that high speed imaging imposes on resolution and contrast of images an in-situ shadowgraph method combined with a sub-pixel accuracy edge detection algorithm have been implemented. The in-situ shadowgraph method does not require applying paint to the specimens eliminating the problems caused by it shattering at high speed. The subpixel edge detection algorithm is able to capture the edge of the specimen within pixels and provides much smoother results when compared to a traditional thresholding algorithm.
The results obtained with this method have been compared against those obtained with more conventional techniques such as video-extensometer and 2D DIC. The results show excellent agreement in the region of uniform deformation, which serves as validation of the proposed technique. When necking appears and the deformation is no longer uniform the results obtained with the conventional techniques are no longer valid while the proposed method is still able to capture the local stress and strain up to fracture.

The method has successfully been applied to characterise stainless steel $304 \mathrm{~L}$ at different strain-rates ranging from $10^{-3}$ to $10^{3} \mathrm{~s}^{-1}$. The quality of the results obtained at the highest speed tested is comparable to that of the lower rates suggesting that the accuracy of the method is maintained at very high speed.

The results obtained applying the proposed technique can be used to improve the calibration of plasticity models including an accurate representation of the strain-rate effect on ductile materials.

We acknowledge AWE for supporting this project and providing expertise that greatly assisted the research.

\section{References}

1. P. Bridgman, Studies in Large Plastic Flow and Fracture (Harvard University Press, Cambridge, Masschusetts, 1964)

2. G.R. Johnson, W.H. Cook, Eng. Fracture Mech. 21(1), 31-48 (1985)

3. G.R. Johnson, W.H. Cook, Proc. of the $7^{\text {th }}$ Int. Symp. on Ballistics, 541-547 (1983)

4. M.G. Stout, P.S. Follansbee, J. of Eng. Materials and Tech. 108(4), 344-353 (1986)

5. R. Smerd, S. Winkler, C. Salisbury, M. Worswick, D. Lloyd, M. Finn, Int. J. of Impact Eng. 32(1-4), 541-560 (2006)

6. E. El-Magd, M. Abouridouane, Int. J. of Impact Eng. 32(5), 741-758 (2006)

7. F.J. Zerilli, R.W. Armstrong, J. of Appl. Phys. 61(5), 1816-1825 (1987)

8. Y. Bai, T. Wierzbicki, Int. J. Of Plasticity 24(6), 1071-1096 (2008)

9. P.S. Follansbee, R.W. Kocks, Acta Metall. 36(1), 81-93 (1988)

10. A. Trujillo-Pino, K. Krissian, M. Alemán-Flores, D. Santana-Cedrés, Image and Vision Computing 31(1), 72-90 (2013) 
